\title{
Guide wire fracture with remnant filament in left circumflex artery and ascending aorta
}

\begin{abstract}
Retained fractured coronary guide wire fragments are rarely encountered during percutaneous coronary intervention, but may cause fatal complication such as thrombosis, emboli, dissection and perforation. Management of retained fractured coronary guide wire depends upon the clinical situation of the patient and the position and length of the fractured guide wire remnant. This article present a case of remnant guide wire filaments that remained in aorta without complications.
\end{abstract}

Keywords: coronary perforation, percutaneous coronary intervention, fractured guidewire, retained guide wire
Volume 10 Issue 6 - 2020

\author{
Kitigon Vichairuangthum ${ }^{1,2}$ \\ 'Division of Cardiology, Suranaree University of Technology \\ Hospital,Thailand \\ ${ }^{2}$ Royal Heart Center, Bangkok, Thailand \\ Correspondence: Kitigon Vichairuangthum, Division of \\ Cardiology, Royal Heart Center, Suranaree University of \\ Technology Hospital,Thailand, Tel +66816144706, \\ Email neoz15@hotmail.com
}

Received: October 26, 2020 | Published: November 16, 2020

\section{Introduction}

Entrapment and fracture of the coronary guidewire is a rare complication of percutaneous coronary intervention (PCI). The incidence of such complication in PCI is reported to be between 0.2 and $0.8 \% .^{1,2}$ But in such cases, life-threatening complications such as embolization, thrombus formation, dissection and perforation may occur. $^{3}$

Although, in most cases, percutaneous retrieval techniques of fractured guide wires are recommended, there have been several reports of fragments being left in place without any complications. ${ }^{4-6}$ This article present the case of a 65 -year-old female patient who was treated with PCI. The patient had remnant guide wire filaments in the left circumflex artery (LCX) and the ascending aorta but did not experience any serious complications during follow up period.

\section{Case report}

A 65- year old female with an underlying disease of hypertension, severe mitral valve regurgitation with history of mitral valve repair operation for over 8 years ago complained of stable angina CCS (Canadian cardiovascular society angina grade) class 3 for 2 months. She also had a past history of coronary angioplasty and one drug eluting stents (DES) were placed in right coronary artery (RCA). Her coronary angiogram show chronic total occlusion at left anterior descending (LAD) artery and significant bifurcation lesion at distal left circumflex (LCX) artery (Figure 1). The patient declined surgical intervention. Considering her decision and symptoms percutaneous coronary intervention (PCI) was planned after an informed consent.

The left main (LM) artery was engaged with a 6 Fr Launcher EBU 3.5 guiding catheter (Medtronic, Minnesota, USA) via the right groin. A 0.014-inch Rinato guide wire (Asahi Intecc Co. Ltd, Aichi, Japan) was passed to distal LCX and another Runthrough NS Extra floppy (Terumo, Tokyo, Japan) guide wire was passed to Obtuse marginal (OM) branch of circumflex. Bifurcation lesion was treated with Crush technique stenting. Predilatation with Minitrek (Abbott Vascular, Santa Clara, CA, USA) balloon size $2.0 \times 12 \mathrm{~mm}$ in both branches, sequentially. And then Biomatrix Alpha (Biosensors, Shangdong,
China) drug eluting stents (DES) $2.25 \times 12 \mathrm{~mm}$ was deployed to OM. The Biomatrix Alpha (Biosensors, Shangdong, China) drug eluting stent (DES) $2.5 \times 30 \mathrm{~mm}$ was deployed, crushing that portion of the $\mathrm{OM}$ stent lying in the main branch (LCX). After the stent implantation, guide wire used to protect the OM branch (Run through NS Extra floppy) could not be removed.

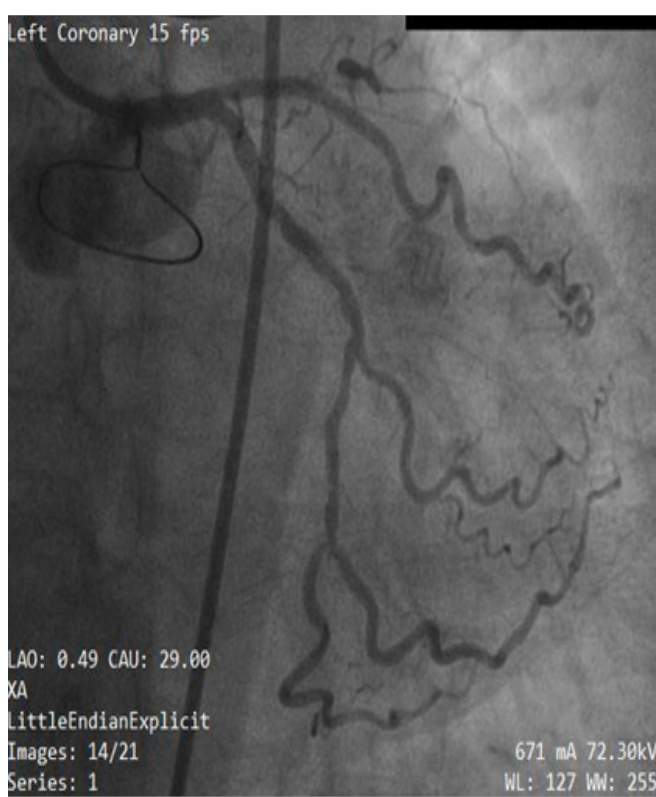

Figure I Initial coronary angiogram of left coronary artery show significant lesion at bifurcation of left circumflex artery (LCX) and obtuse marginal 2(OM2) branch.

Subsequently, we made several attempts using simple manoeuvers like traction, pull and push without success. Unfortunately the guide wire was fractured and entrapped leaving part in LCX and LM (Figure 2). The patient suddenly complained of severe chest pain. The blood pressure went down to 70/50 $\mathrm{mmHg}$. The fluoroscopic test injection show severe LM (Figure 3) and left circumflex (LCX) artery dissection (Figure 4). Due to rapid hemodynamic destabilization, Subsequently, LM stenting with $3.5 \times 18 \mathrm{~mm}$ Biomatrix Alpha drug 
eluting stent (Biosensors, Shangdong, China) was performed (Figure 5). Another Biomatrix Alpha (Biosensors, Shangdong, China) drug eluting stent (DES) $2.75 \times 18 \mathrm{~mm}$ was successfully crossed with the broken guidewire and was deployed at LCX to sealed the fractured fragment to the vessel wall.The procedure result was excellent with Thrombolysis in Myocardial Infarction (TIMI) 3 flow distally (Figure 6). After the placement of the stents, an intra aortic balloon pump (IABP) was placed. The patient's chest pain was resolved. The hemodynamic condition was improved but we later observed retained filaments in the ascending aorta (Figure $7 \& 8$ ). The patient declined surgical intervention for removal of these stray filaments and was discharged the following day with stable condition. During the subsequent monthly follow up visits, she was in a good condition and follow up with triple anti-platelet medication with no complications.

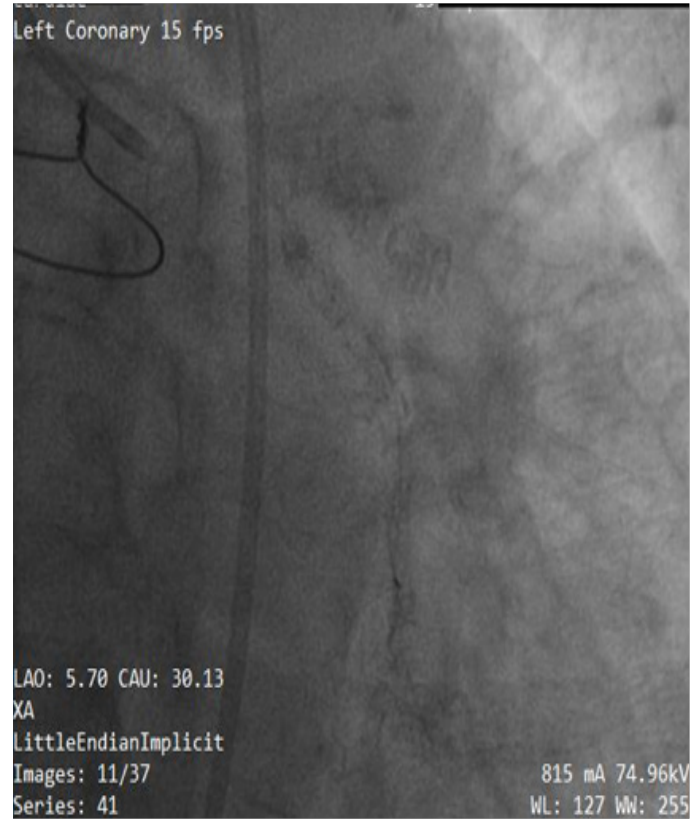

Figure 2 After deployment of the stents over the left circumflex artery (LCX), a fracture occurred at the LCX and left main (LM).

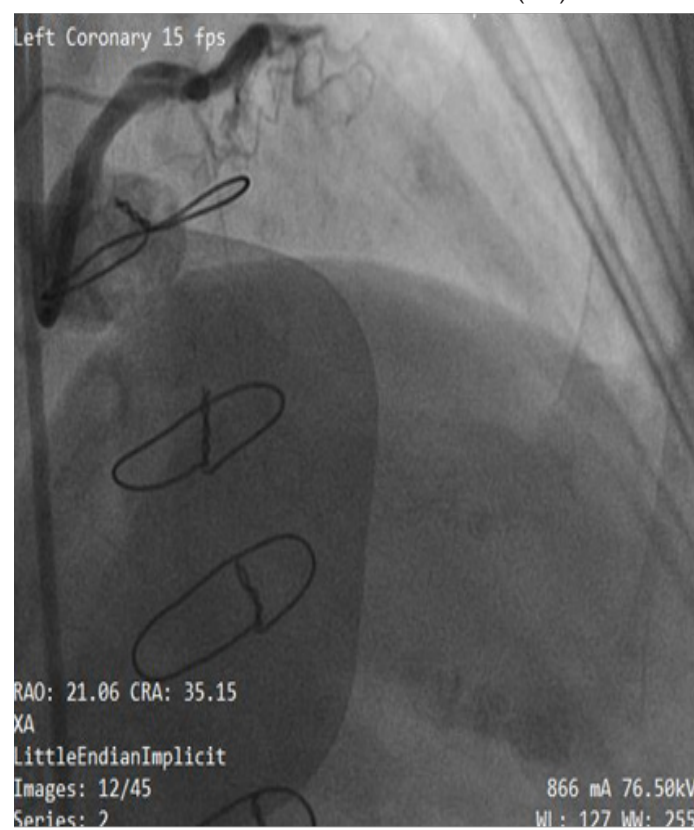

Figure 3 Angiography confirmed the left main (LM) dissection.

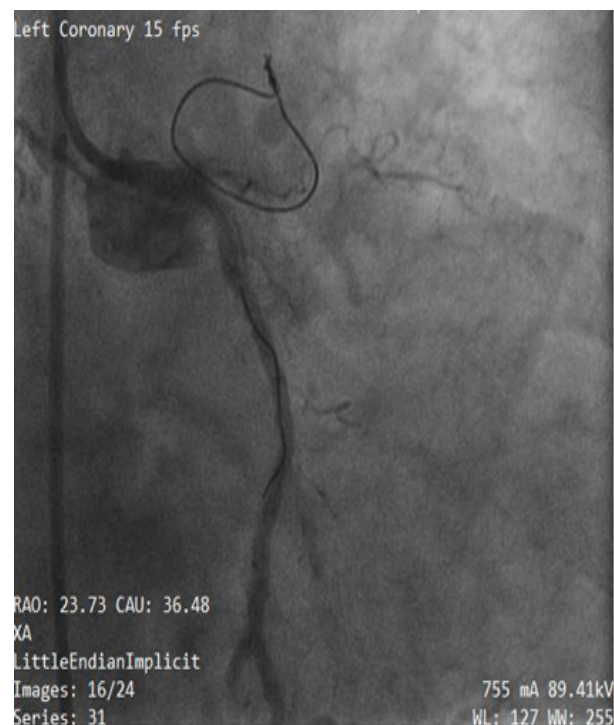

Figure 4 Angiography confirmed the left circumflex artery (LCX) dissection.

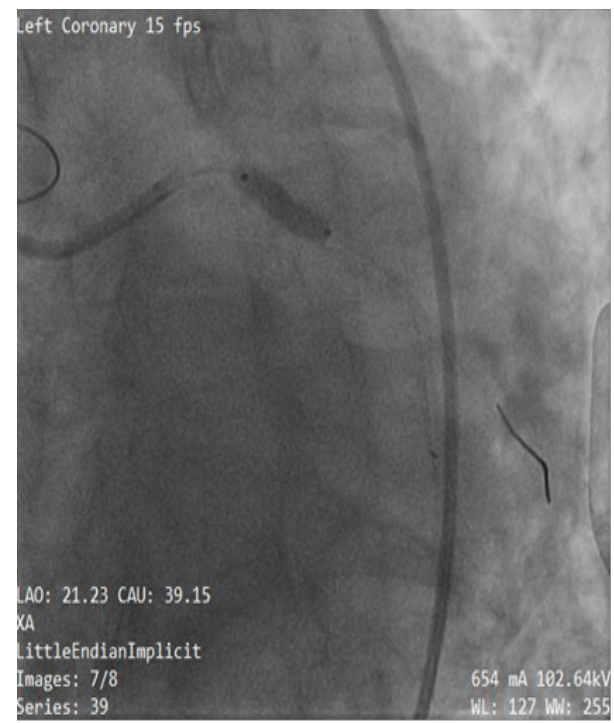

Figure 5 Attempt $\mathrm{PCl}$ of $\mathrm{LM}$.

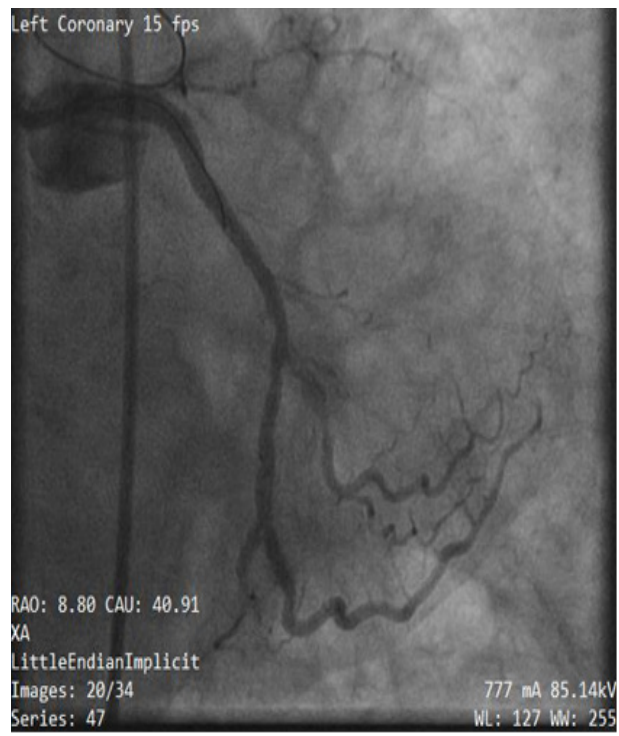

Figure 6 Good TIMI flow after stenting. 


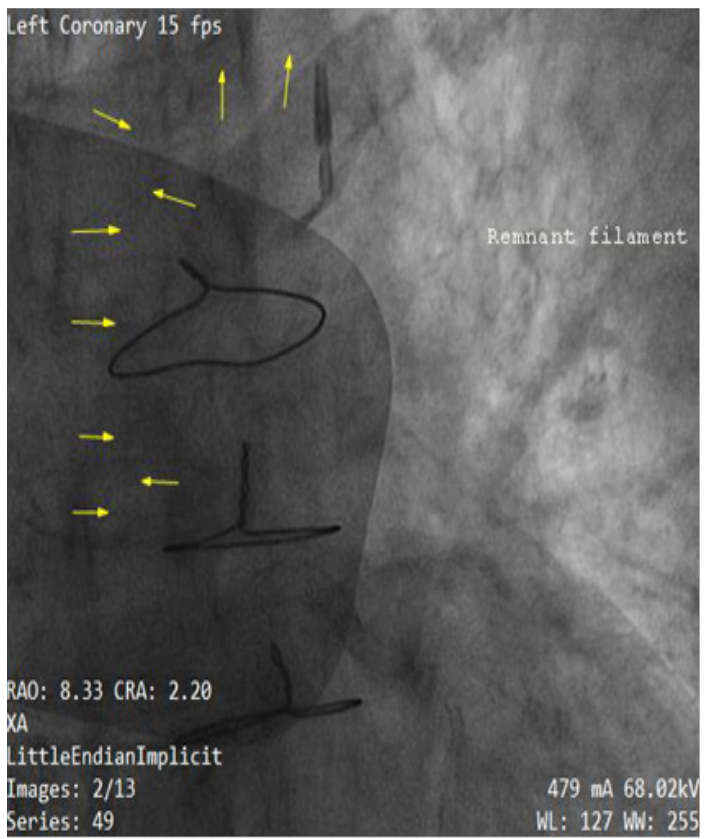

Figure 7 Retained filaments in ascending aorta.

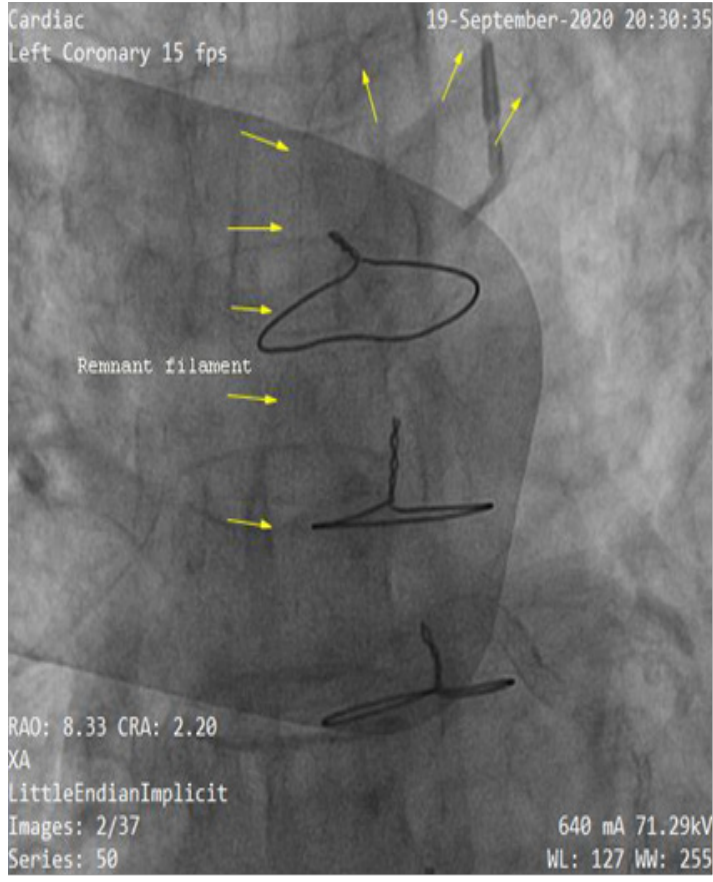

Figure 8 Retained filaments in ascending aorta.

\section{Discussion}

Guide wire fracture and entrapment is a rare but serious complication during percutaneous coronary intervention (PCI). The possible mechanics of the rupture entails several factors: Excessive rotation especially if the tip is not free, ${ }^{7}$ Complex and bifurcation lesions stenting, multiple usages of the same guide wire, ${ }^{8,9}$ excessive bending while torque produces a high tensile load to the guide wire which increases the risk of guide wire fracture and entrapment in the coronary vessel, ${ }^{10}$ Despite technical improvements and development of more flexible and high-quality guide wires, the incidence of these complications is increasing. ${ }^{11}$ Retained fractured coronary guide wire fragments may cause fatal complication such as thrombosis, emboli, dissection and perforation. There are several methods recommended for the management of fractured guide wires, including snare removal, ${ }^{12,13}$ two- or three-wire rotation, ${ }^{14}$ stenting over the retained wire, ${ }^{10}$ and conservative treatment. ${ }^{4-6}$

In the present case, guide wire was entrapped between stent and a calcified coronary plaque and broke after forceful attempts to remove. Guide wire fracture with remnant filament complicated with LM and LCX dissection. Ways of avoiding such a complication like wire removal from the side branch before main branch stent placement, guide disengagement while pulling on the entrapped wire or microcatheter/balloon use to reduce necessary pulling forces should be done. Even though Surgical extraction is strongly recommended in cases of protrusion of the guide wire into the ascending aorta,, 15 this patient was conservative treatment and stented over the retained wire to LM because she refused surgical intervention. To eliminate the guide wire fragment from the lumen and reduce the risk for coronary thrombosis, sealing the fractured fragment to the vessel wall with the use of stents was described after unsuccessful retrieval of the retained fragment in the vessel, vascular endothelial cell covering over the guide wire fragments may render them immobile and nonthrombogenic. ${ }^{16,17}$

Management of retained fractured coronary guide wire depends upon the clinical situation of the patient and the position and length of the fractured remnant. In a desperate situation, the operators should be aware of this complication and be familiar with the measures to avoid it and to appropriately manage it.

\section{Conclusion}

In cases when percutaneous retrieval has failed, surgical extraction of the remnant fragments is recommended. Albeit the most ideal management for remnant guide wires is their removal, conservative treatment with the fragments left in situ may be successful in some cases in which patients remain asymptomatic with hemo-dynamically stable. However, life-long administration of intensive anti-platelet medications and close observation are recommended for these patients.

\section{Conflicts of interest}

The author declares no conflict of interest.

\section{Acknowledgments}

None.

\section{Funding}

None.

\section{References}

1. Kaplan S, Kaplan ST, Kutlu M. An unusual case of guide wire fractured during primary percutaneous coronary intervention and two year follow up. Kardiol Pol. 2010;68(11):1291-1293.

2. Balbi M, Bezante GP, Brunelli C, et al. Guide wire fracture during percutaneous transluminal coronary angioplasty: possible causes and management. Interact Cardiovasc Thorac Surg. 2010;10(6):992-994.

3. Abdulrahman M, Al-Moghairi, Hussein SA. Management of Retained Intervention Guide-wire: A Literature Review. Current Cardiology Reviews. 2013:9(3);260-266. 
4. Cafri C, Rosenstein G, Ilia R. Fracture of a coronary guidewire during graft thrombectomy with the $\mathrm{X}$-sizer device. J Invasive Cardiol. 2004;16(5):263-265.

5. van Gaal WJ, Porto I, Banning AP. Guide wire fracture with retained filament in the LAD and aorta. Int J Cardiol. 2006;112(2):e9-11.

6. Hong YM., Lee SR. A Case of Guide Wire Fracture With Remnant Filaments in the Left Anterior Descending Coronary Artery and Aorta. Korean Circ J. 2010;40(9):475-477.

7. Ghosh PK, Alber G, Schistek R, et al. Rupture of guide wire during percutaneous transluminal coronary angioplasty. Mechanics and management. J Thoracic Cardiovasc Surgery. 1989;97(3):467-469.

8. Singh D, Darbari A. Retrieval of trapped and broken guide wire with immediate rescue off-pump coronary bypass surgery. Interact Cardiovasc Thorac Surg. 2014;19(3):529-531.

9. Coskun E, Altınay L, Tekin A, et al. Entrapment of Broken Guidewire in the Coronary Artery: A Rare Percutaneous Coronary Intervention Complication Requiring Urgent Revascularization. International Journal of Cardiovascular Sciences. 2020;33(4):419-422.

10. Naeem MM, Mujtaba SF, Saghir T, Zeb S. Broken Coronary Guide Wires In Coronary Tree: A Simple, Safe And Cost Effective Technique For Successful Retrieval. Pak Heart J. 2016;49(03):195-199.
11. Singh D, Darbari A. Retrieval of trapped and broken guide wire with immediate rescue off-pump coronary bypass surgery. Interact Cardiovasc Thorac Surg. 2014;19(3):529-531.

12. Hewarathne, Kularatne, Karunarathne et al. Guide wire Fracture, Entrapment in Proximal Left Anterior Descending and Successful Extraction with Snare Catheter. Interv Cardiol. 2019;11(3):61-65.

13. Kim TJ, Kim JK, Park BM, et al. Fatal subacute stent thrombosis induced by guidewire fracture with retained filaments in the coronary artery. Korean Circ J. 2013; 43(11):761-755.

14. Kume K, Yasuoka Y, Sasaki T. Successful percutaneous retrieval of a guiding catheter tip that had unexpectedly become detached using a twowire technique. Int J Angiol. 2016;25(5):160-162.

15. Kilic H, Akdemir R, Bicer A. Rupture of guide wire during percutaneous transluminal coronary angioplasty, a case report. Int $J$ Cardiol. 2008;128(3):e113-e114.

16. Lopez-Minguez JR, Davila E, Doblado M, et al. Rupture and intracoronary entrapment of an angioplasty guidewire with the $\mathrm{X}$-Sizer thromboatherectomy catheter during rescue angioplasty. Rev Esp Cardiol. 2004;57:180-183.

17. van Gaal WJ, Porto I, Banning AP. Guide wire fracture with retained filament in the LAD and aorta. Int J Cardiol. 2006;112(2):e9-11. 